\title{
A video-based assessment of likeability and usability in a play application of robots for children
}

\author{
Maria Luce Lupetti, Maria Franca Norese, Xiaolu Wu and Haipeng Mi
}

Maria Luce Lupetti is based at the Faculty of Industrial Design Engineering, Delft University of Technology, Delft, The Netherlands. Maria Franca Norese is based at the Department of Management and Production Engineering, Politecnico di Torino, Turin, Italy. Xiaolu Wu is based at Politecnico di Torino, Turin, Italy. Haipeng Mi is based at Tsinghua University, Beijing, China.
Received 21 November 2018 Revised 9 January 2019 Accepted 16 March 2019

(C) Maria Luce Lupetti, Maria Franca Norese, Xiaolu Wu and Haipeng Mi. Published by Emerald Publishing Limited. This article is published under the Creative Commons Attribution (CC BY 4.0) licence. Anyone may reproduce, distribute, translate and create derivative works of this article (for both commercial and non-commercial purposes), subject to full attribution to the original publication and authors. The full terms of this licence may be seen at http://creativecommons.org/ licences/by/4.0/legalcode

\begin{abstract}
Purpose - The purpose of this paper is to conduct research with children, who have different abilities from adults, in terms of language understanding and level of attention, is a challenging task, especially concerning novel interactive systems such as social robots. Consequently, self-reporting methods are often replaced or supplemented by observational methods that are usually carried out taking advantage of video recordings. However, some limitations make this approach challenging for studies conducted with groups of children in real-world environments, whose relevance is being addressed more and more frequently in human-robot interaction (HRI) research. Thus, there is a growing need for rigorous observation approaches in unstructured test environments.

Design/methodology/approach - This paper presents an alternative analysis approach, in relation to an experimental child-robot interaction (CRI) application, which was developed at the Academy of Arts and Design, Tsinghua University, China. The proposed methodology is based on the analysis of video recordings of in-wild activities of children with a robot. The methodology has the aim of providing a framework to facilitate knowledge identification and structuring. It was implemented for experiment evaluation and validation purposes and to propose a reference structure for the organization of new experiments and the stimulation of new ideas and activities in the design process.
\end{abstract}

Findings - This methodology provides a logical structure, which can be used to identify the effectiveness or limits of design choices, pertaining to such aspects as the morphology or movement of robots or the choice of their specific role in education, all of which play crucial roles in the design process and could be improved to achieve better results. This structured identification is a practical implication for the design process, above all when it is oriented toward social robots and their interaction with children or elderly senile people. In this case, the outcomes were the identification of important elements of an experiment (psychological profiles of the involved children and possible problems or risks) and their impact on the design process.

Originality/value - The methodological approach, which structures and uses cognitive maps to elaborate multicriteria evaluation models, is not new to the operations research field (where it is defined as a multimethodology application of Soft OR), but it has not yet been applied in the field of HRI studies, to analyze children's perception of a robot and to identify the factors that can affect a good CRI or to structure knowledge that can be shared to guide the design process of robots for the experience of children playing.

Keywords Cognitive mapping, Child-robot interaction, Knowledge acquisition and structuring, Multicriteria modelling, Social robot design

Paper type Research paper

\section{Introduction}

Conducting research with children is a challenging task, especially when dealing with novel interactive systems, such as social robots. It requires an adaptation of the methods, especially in evaluation studies. Children are, in fact, characterized by different abilities, compared to adults, in terms of language understanding, level of attention (Hanna et al., 1998), developmental factors and temperamental factors (Read and MacFarlane, 2006). As 
a consequence, self-reporting methods are often replaced or supplemented by observational methods because of their unobtrusive nature (Robert and Breazeal, 2012).

Although the challenges of evaluation studies with children have already been addressed by some authors within the child-robot interaction (CRI) community, Kennedy et al. (2015) and by Charisi et al. (2016), there are still no consolidated methodologies available to assess some perceptual aspects of robots, such as animacy, anthropomorphism and likeability. These methodological challenges are becoming even more prominent in the case of studies conducted in the wild, whose relevance is being addressed more and more in human-robot interaction (HRI) research (Baxter et al., 2016). When conducted as applications in real environments, the studies are in fact characterized by an unconstrained interaction nature, which results in the impossibility of categorizing the participants' behavior a priori (Feil-Seifer and Matarić, 2011).

This lack of structured observation methodologies, and the fact that video-based analysis can be a very laborious activity (Bartneck et al., 2009), point out the need for novel approaches to extract meaningful knowledge from unstructured test conditions.

Thus, a knowledge structuring approach, based on cognitive mapping for the elaboration of multicriteria evaluation models, was identified as a potentially effective way of conducting a behavior analysis based on video recordings.

Although such an approach is not new to the operations research field (where it is described as a multimethodology application of Soft OR), it has not yet been applied within the field of HRI studies.

The proposed approach was applied to analyze of children's behavior, which was recorded during a play activity with a low-anthropomorphic social robot called Shybo.

\section{Evaluation of the play activity with Shybo}

Shybo (Lupetti, 2017) and its related activity are the results of a design research project that was developed at the Department of Architecture and Design, Politecnico di Torino (Italy). This research, which was carried out adopting a research through design approach (Frayling, 1993), combined participatory actions with the physical development of a prototype and its accessories. The abilities of the robot consist of perceiving sounds and reacting by lighting up, in different colors, and of acting through minimal nonverbal behavior, namely, movement of its hat. It was designed to be part of experiences aimed at fostering children's reflections, which may be carried out in a class. One application of Shybo refers to an experiment that was carried out at a primary school in China, as part of a collaboration with the X-Studio (Tsinghua University). This experience involved 12 children and lasted about $2 \mathrm{~h}$.

Feedback and insights have already been collected to evaluate the soundness of the solution and to acquire reusable knowledge about how to design acceptable child-robot play experiences (Lupetti et al., 2017). The data collection consisted of the video recordings of the experiment, the children's feedbacks through a questionnaire and the results obtained from two assessment forms, elaborated by referring to Read et al. (2002). These were used to evaluate the children's perceptions of difficulty in the activities of the experiment and to establish their willingness to do the experiment again.

The data collection methods were defined by referring to related literature on interaction design for children and CRI studies. As reported by Charisi et al. (2016), the methods used to evaluate the interaction of children with novel interactive systems can be summed up as self-assessment methods, behavioral observations, psycho-physiological metrics and task performance measurements. Among these, self-assessment and observational methods were selected as being the most relevant. In fact, given the playful and open nature of the 
activity, the measurement of a task performance was not relevant for the study, and the use of devices to detect psycho-physiological metrics might have affected the experiment.

The questionnaire and the forms resulted in answers very similar from each child, thus, these were considered unreliable and excluded from the evaluation. This was probably due to the fact that the concepts under evaluation were too abstract for children of this age, and to the fact that the questionnaire and the forms were submitted to the children in the group, rather than to individuals. This caused distraction and discussion among children about what answers they were giving.

Therefore, the videos of the experiment became the crucial source of knowledge. In the preliminary analysis, the videos were coded manually using an open-source tool for behavioral observation, called Boris, with the aim of identifying several recurring and relevant forms of behavior that would be useful for the subsequent analysis. These were then used to assess the children's engagement and their enjoyment of the experience and the attractiveness of the robot. However, this first analysis was affected negatively by some limitations, above all concerning the difficulties of observing a group interaction in a real context and the absence of a complete transcription and translation in English of the children's comments. The study was, in fact, carried out as part of a brief collaboration with a Chinese research group, but designed by a non-Chinese speaking researcher. The absence of a complete transcription of the children's comments, therefore, represented a crucial limitation to the production of reusable knowledge that could offer information on the design of novel robots and their related activities for children.

Given these limitations, a further analysis on the video-recording was carried out by a multidisciplinary team that included a Chinese speaking investigator. The aim of this novel study was to generate knowledge that combining the video observations with an analysis of children's comments, would provide useful insights and guidance in the design process for future robot applications for play, aiming at a more efficient approach to the analysis of the video recordings.

This analysis and evaluation approach can be considered as a methodological proposal that could be useful for similar situations, in which different non-quantitative elements of knowledge have to be structured and used to facilitate decision and action. This methodological approach has been used in several contexts (Norese, 2011; Norese et al, 2015). In this case, it was tested to verify or improve the quality of the previous requirements, to use them in the design process and to propose a new analysis framework for the HRI research field, when social robots have the purpose of promoting the social engagement of children.

Thus, Section 3 describes the knowledge acquisition process and a methodological approach. This is followed by the results of the analysis of the activity carried out with Shybo in Section 4. The analyzed system included not only the children but also their parents, tutors and non-human actors, such as a robot, cameras and a TV, which activated the words, impressions and actions of the human actors. Finally, Section 5 concludes the paper.

The experiment consisted of the following four phases (Figure 1):

1. Introduction to musical instruments, the creation of groups of children and choosing a color for each group.

2. Video presentation of the robot plus first visual contact with it and training of the robot group by group.

3. Introduction of the color theory and explanation of the game rules.

4. Playing the board game and, eventually, the involvement of the parents in playing the game.

The interaction between children and a robot started in the second phase, in terms of the first visual contact and the children's reactions to the behavior of the robot during the training 

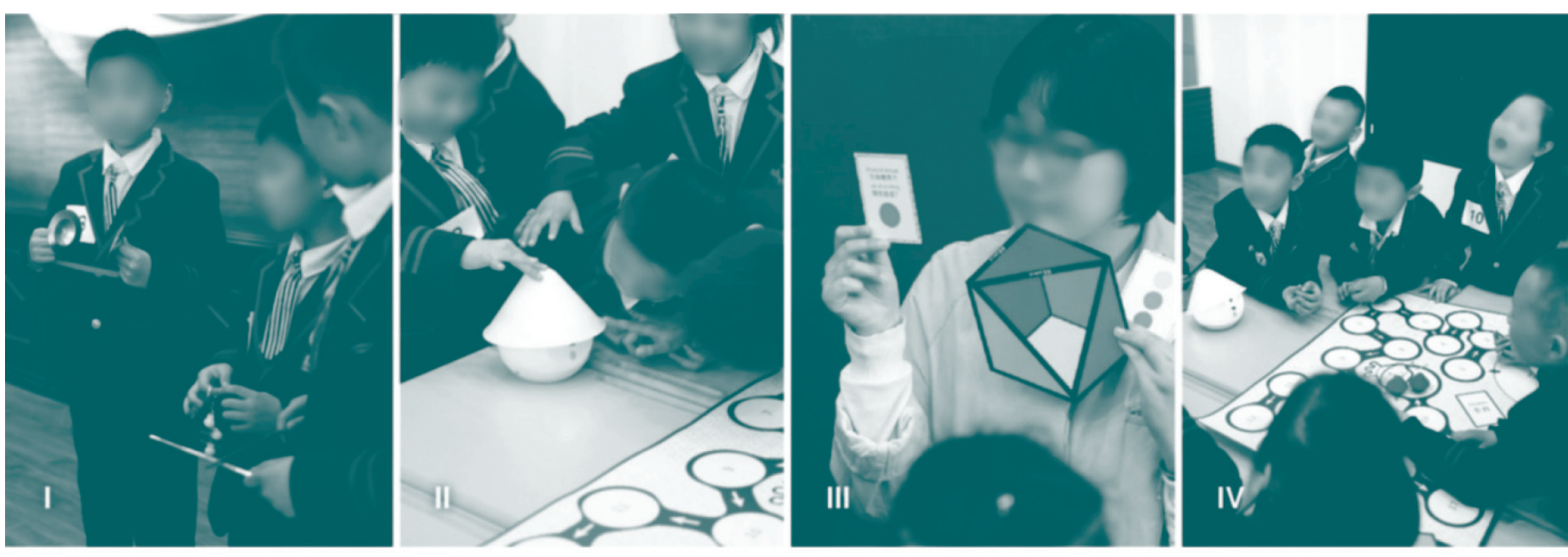

Notes: (a) Selection and grouping of musical instruments; (b) familiarization with the robot; (c) introduction to key concepts tof color theory; (d) play with a board game and the robot

activity, and then developed into engagement in the game in the last phase. In the first and third phases, the interaction between the children and the robot was limited or absent.

\section{Knowledge acquisition and structuring}

The methodology consists of a structured process of knowledge acquisition and use to facilitate decision, in this case in relation to a design process.

A knowledge base was created by means of repeated visualizations of the video recordings by the Chinese investigator (who was not involved in the design phase) and her analysis of some critical parts of the videos with the other author, who designed the experiment and who played a role as an actor in the system for the current study. The result was the transcription and translation of the actors' verbal expressions, plus a description of the facial expressions and behavior of 12 children, their parents, the Chinese and Italian tutors and a photographer, in relation to technologies (the robot, cameras and tools used in the game), which can be considered the non-human actors of this system (Law, 2008).

Five sets of factors are proposed in the literature to evaluate HRI (Bartneck et al., 2009), but they are above all oriented toward analyzing adults who interact with robots in production processes. The consistency of these factors with this specific child-related robot application was tested by means of a structured reading of the text and identification and distinction of elements in relation to the five sets of factors. Three sets of factors were recognized as not being consistent with the context, while the likeability of a robot emerged as one of the most significant aspects, above all in the second phase of the experiment; usability was identified as the most significant aspect of the quality of the designed experiment.

At the same time, this analysis underlined the different kinds of behavior of the children and the need to identify their different profiles of engagement in the experiment, to deal with the concepts of likeability and usability. A tree was used to represent the conceptual structure of a model, where the two main branches (Figure 2) were the aspects that had to be taken into consideration: usability of the experiment in other experimental contexts and in educational practice; and likeability, an aspect that can include the quality elements of the interaction between children and a robot. The leaves of the tree were not clearly defined in the first model structure because they were only included as a suggestion of what a deeper 


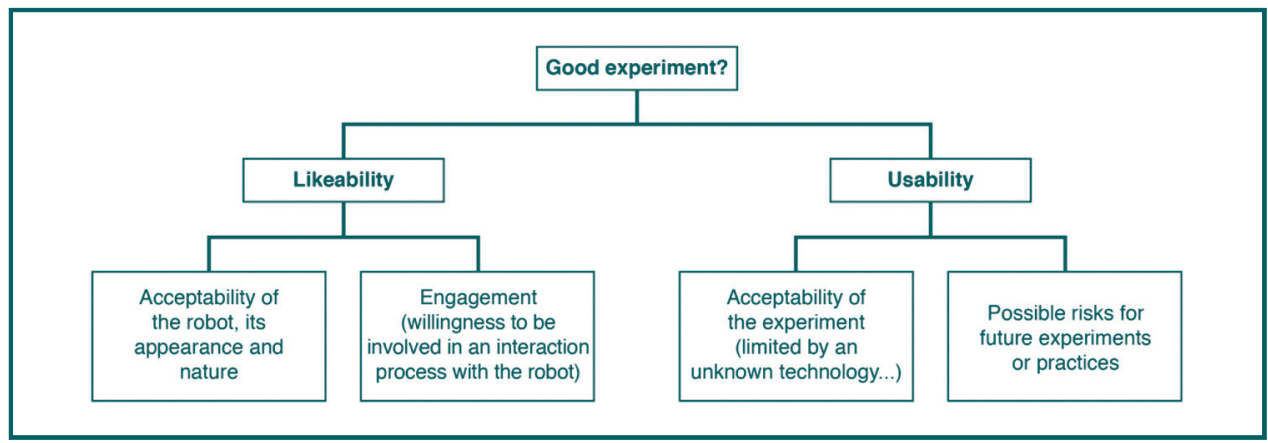

analysis of the knowledge elements could infer from extracting, combining and studying elements from the coded text, in relation to the main aspects, that is, likeability and usability.

This more focused analysis produced a list of actions and the verbal or physical expressions of each child, in terms of initial and subsequent impressions, interaction with the robot and the other involved actors, curiosity, empathy, etc. A cognitive mapping approach was activated to structure these confused elements and to translate them into the conceptual structure of a multicriteria model and then into criteria (analytic formalization of the conceptual aspects) to evaluate the quality of the experiment and to suggest improvements for the design process.

\subsection{Cognitive mapping}

A cognitive map can be used to construct and accumulate knowledge; thus, allowing the "mind's eye" not only to visualize images or physical paths but also the structure that links concepts in terms of specific relationships, such as causality, influence, explanation, complementarity, contradiction, etc. A cognitive map is a network in which nodes indicate concepts and arcs denote relationships between concepts. Each concept can be associated with a proposing source and characterized by a typology, and the relationships have to be coded to distinguish their meanings (Huff and Jenkins, 2002; Eden, 2004; Norese and Salassa, 2014).

Two kinds of map were created, the first to represent the different types of behavior of the children (and then to identify their possible profiles) and the second to cluster concepts that presented a certain proximity and to connect them, to analytically express the component elements of likeability and usability. The knowledge elements (actions, words and expressions of the involved actors, such as surprise, excitement or disappointment) that were acquired from the video analysis were included in nodes, as a textual copy of the expressed words or descriptions of the actions and physical expressions. The associated actor's code, the experiment phase and a conceptual synthesis of the words were indicated in brackets near the words in the nodes.

Relationships between the nodes can be identified to reduce the complexity that can be present in a concept (multiple meanings, ambiguity and difficult distinction between apparent or real contradiction of other concepts), to facilitate a clear, structured and transparent interpretation. In this case, only three kinds of relationship were used: cause-effect, in agreement with and contradiction.

A map that synthesizes the different verbal expressions of Child No.10 is presented in Figure 3, while Figure 4 includes the different expressions of the children in relation to the Likeability aspect. 


\section{Figure 3 Verbal expressions of a child in the experiment}

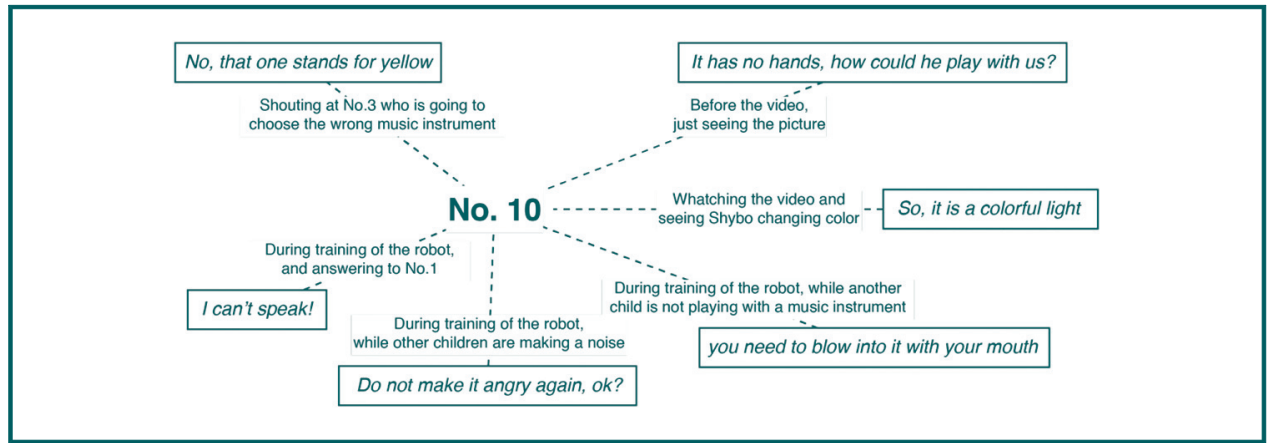

\section{Figure 4 The cognitive map on the Likeability aspect of the robot}
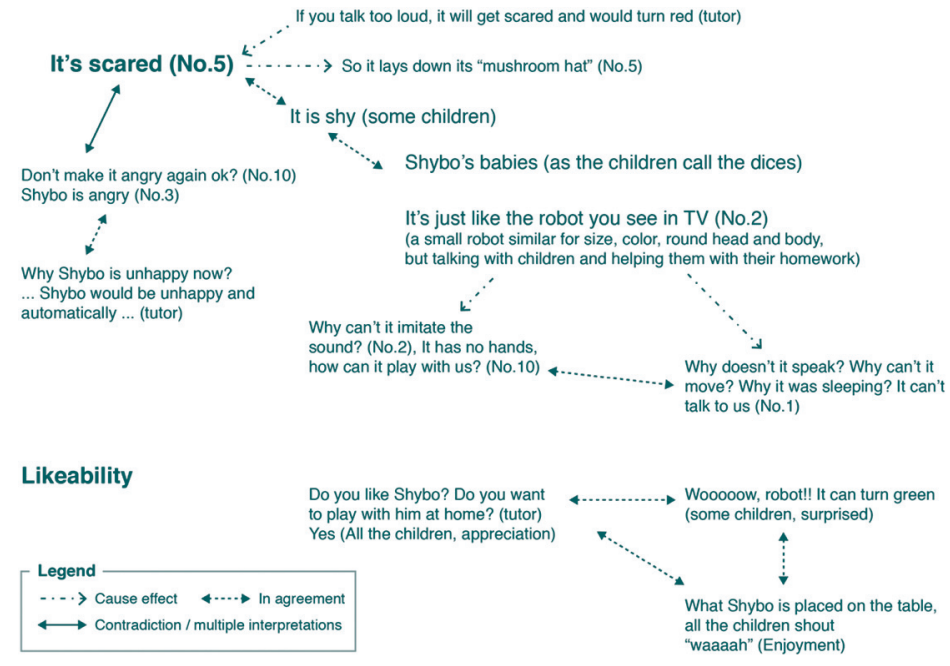

\section{Results}

\subsection{The children's profiles}

The interactions of the children with the robot and, in general, with the experiment, resulted to be different among the children, both in the videos and in the structured syntheses of knowledge from the videos (the lists of actions and verbal or physical expressions of each child and the cognitive maps where these elements were logically connected to understand the meanings of some not so clear reactions).

These knowledge structures stimulated an analysis of the different profiles of the children, which was conducted to facilitate the likeability analysis and to acquire elements that could facilitate the repeatability of the experiments in other contexts and in practice. There was no interaction between the children and the robot in the first phase, but it was possible to define some children's profiles (for example, the children who helped others to learn how to play the musical instruments were considered experiment facilitators). The interaction between the children and the robot was also very limited in the third phase, while the second phase was different, and the descriptions of the impressions and actions of the children in this phase were used to facilitate the definition of the profiles, together with an 
analysis of the children's reactions to the game presentation and activation, to consider their active or passive attitudes of being involved and to involve their parents in the game (Phases IV and V).

A first distinction was made between "strong" and "weak" children. A child who clearly understood the process and showed the capability of following and reacting was considered strong. Weak refers to those children who not only had difficulty in understanding the situation and the procedure (mental weakness) but also limited engagement in the experiment. The result mostly due to certain specific characteristics of a child, such as timidity or habitual lack of attention. Some children clearly understood everything and demonstrated acceptability and adaptability, others were very curious about the nature of the robot that they wanted to know better and about other involved technologies. Some children asked questions and carefully organized their groups during the game.

The profiles of these strong children were different from each other and, for these reasons, another distinction was implemented (Table I). In this table, four categories of children are described in terms of their different types of behavior in the interaction process with the tutors and during the game. Active means posing and answering questions, following the tutor's instructions, understanding the situation, explicitly associating specific elements of the experiment with their previous experience. Engaged means the capability of unconsciously passing from a simple role to another more mature role: from the role of follower (participating in the game and sometimes asking questions) to the facilitator role (explaining things to others, verbally stimulating other children or a tutor, calming some others) and, finally, to the organizer role (organizing their groups well, instructing their parents or validating their parents' activity). Some children participated in the game but did not demonstrate any form of transition from one role to another. Only one child (No.7) participated in the game without showing any curiosity about the robot, game or experiment, and always focused attention on an unknown musical instrument. The star indicates a mixture of mental weakness and timidity, which was present in 3 of the 12 children.

\subsection{From some cognitive maps to a multicriteria evaluation model}

The definition of these profiles facilitated the activity of acquiring and structuring new knowledge elements to complete, formalize and validate the evaluation model.

The first result was a list of problems and possible risks for future experiments, which were identified and analyzed because they seemed to be interesting for both the design process and the usability component of the evaluation model. Other important results were the use of the knowledge elements pertaining to the children's willingness to be engaged in the experiment, to analyze the acceptability of the experiment aspect and the increase of these knowledge elements to include other factors and deal with the likeability of the robot aspect. The analyzed elements were structured in four cognitive maps, in relation to the likeability of the robot (Figure 4), interest and engagement in the experiment, evolution of the children's role and curiosity concepts, which were used to re-define the model structure and elaborate a set of evaluation criteria.

\section{Table I Engagement profiles}

Children (coded name)

No.2, No.3, No.5, No.10, No.9

No.1, No.6

No.4, No.8, No.12, No.11

No.7
Engagement profiles

Active in the interaction process with the tutors (in each phase of the experiment) and engaged in the game (Phases IV and V)

Only engaged in the game

Only participating in the game

Distracted

\begin{tabular}{l|l|l} 
PAGE 116 & MEASURING BUSINESS EXCELLENCE & VOL. 23 NO. 22019
\end{tabular} 
The cognitive map in Figure 4 includes several words that express the empathy that emerged in the interaction of the children with the Shybo robot, which is described as shy, angry or scared, and the dice of the game that were defined by the children as Shybo's babies. All the children liked Shybo and were surprised when it changed color the first time. Other interesting aspects concern the children's interest in the nature and functions of the robot and the clear and immediate association between Shybo and a small robot they had seen on TV, which was similar in size, color and head and body shape, but which talked to the children and helped them with their homework.

The analysis of these reactions produced a proposal of dealing with the likeability of the robot aspect considering two different criteria, mental engagement, in terms of percentage of children interested in the nature and functions of the robot; and emotional engagement, which was expressed as the percentage of children that demonstrated a willingness to be involved in an interaction process with the robot, through expressions of empathy and acceptability.

The usability of the experiment in educational contexts aspect includes acceptability of the experiment and control of the risks that reduced the acceptability of the experiment or which could affect future experiments or practices. Acceptability dealt with two criteria:

1. engagement in the game, in terms of unconscious evolution of the children's roles; and

2. acceptability, in terms of willingness of the children, to be involved in an interaction process with the tutors and curiosity, in relation to each technology that could have played a role in the experiment.

These criteria were expressed by the percentage of children.

The risk aspect dealt with four criteria (technical risks, complexity of the experiment, components that confused or distracted the children and components that were time consuming) that were used to evaluate the severity of the different risks associated with each phase and the options of risk control that could be activated.

The components of this model can be used separately to evaluate likeability or acceptability or to evaluate the risk component and its present or future impact. Another possible approach synthesizes all the components (and criteria) by means of a multicriteria method (Roy, 1996) that accepts both quantitative and qualitative data to generate evaluations. The method can be used to evaluate the overall quality of the experiment and compare it with other experiments, to compare the quality of the different phases or to compare the overall or phase quality with a monitoring reference model, to use this information in the design process (Figure 5).

\subsection{Implications for practice and future research}

This process of knowledge acquisition and use produced a sequence of results, which have different practical implications. The different psychological profiles of the children, when interacting with the robot in a playful context, can be used to better define the role of robots in education (O'Keefe, 2016), and, at the same time, they facilitate the definition of a multicriteria evaluation model (Norese, 2016). The identification of some problems, during the experiment, stimulated a risk analysis and the elaboration of some risk control options, which could be useful in the design process. At the same time, these elements were used to evaluate the overall quality of the experiment.

The results of this study have also implications for future research, both for the human-robot interaction field and for operations research field. In fact, on the one hand, although a knowledge structuring approach is not new to the operations research field it has not yet been applied within the field of HRI studies. This is particularly useful because of the growing need of methods and approaches for dealing with unstructured testing environment and qualitative analysis. On the other hand, because of this uncommon relationship between the two fields, this case study contributes to providing an example of 


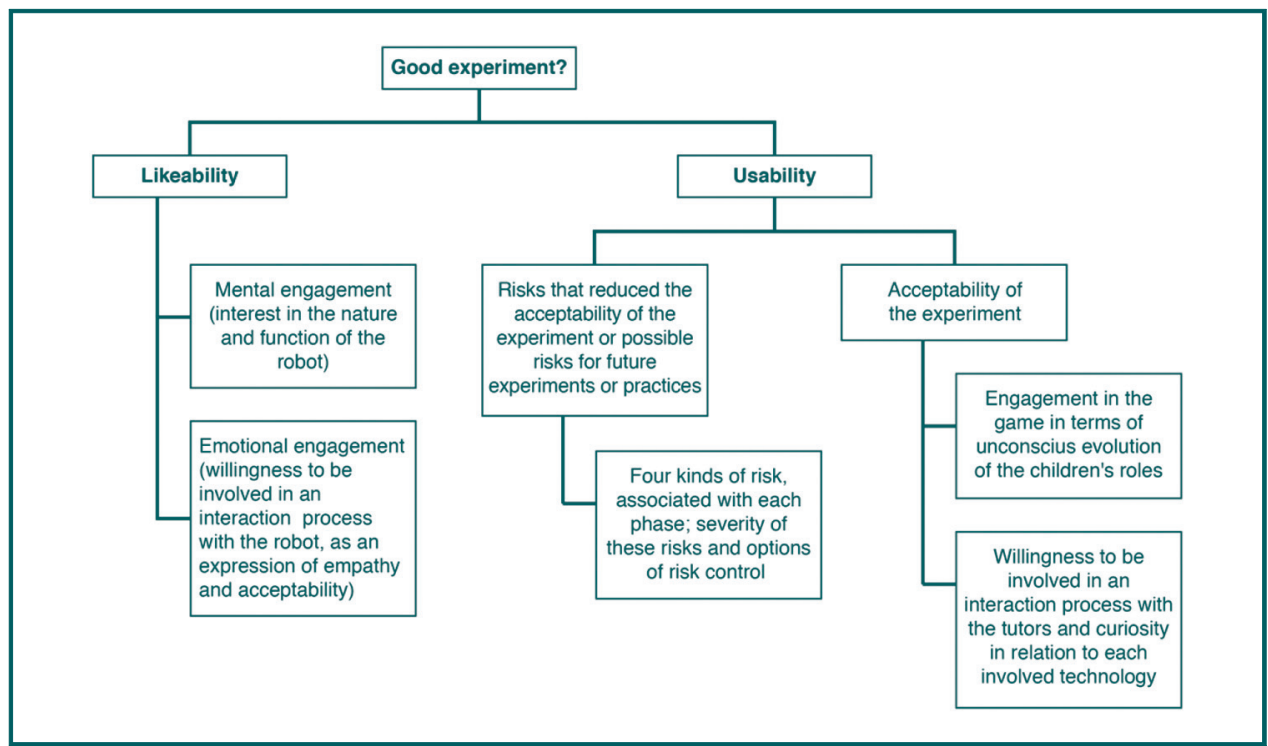

how and why operations research might be relevant and helpful in HRI studies, pushing the boundaries of its application areas.

\section{Conclusions}

HRI studies are facing more and more complex challenges, especially within particular contexts of applications that imply the engagement of participants with very peculiar characteristics, such as children. Furthermore, there is a growing interest for studies in a real environment that might result in less structured and rigorous than inlab studies.

This is calling for novel methods and approaches to address such less structured nature of studies and the peculiar nature of participants. Among various methods, observation and analysis of video recordings are becoming a common practice. However, some limitations, such as dealing with groups and subjectivity in the observation approach, make this approach challenging.

Because of this growing need for rigorous observation approaches in unstructured test environments, we presented an alternative analysis approach, in relation to an experimental child-robot application, which was tested out at a primary school in China. Our approach provides a logical structure, which can be used to identify the effectiveness or limits of design choices, pertaining to such aspects as the morphology or movement of robots or the choice of their specific role in education, all of which play crucial roles in the design process and could be improved to achieve better results.

The presented analysis has produced important knowledge for the design process of sociotechnical systems. Knowledge identification and structuring have been facilitated by an approach that focuses on the children's mental and emotional engagement, and, at the same time, on their process of involvement in a logic of learning through play.

Marginal and not so marginal uncertainties that are associated with previous design choices have been identified using the knowledge acquired through experiments and have been structured in terms of risk assessment and control. This approach is possible when the design process accepts the idea that needs and requirements are not completely and clearly defined at the beginning of the process, but have to be identified during the 
evolution of the design process and used to enrich the understanding of the challenges our society will have to face in the near future. This is particularly suitable and desirable in processes that adopt a research through design approach, in which it is central to iterate and continuously reframe the issue addressed.

Despite the constructive results and the potentials of the proposed approach, this presents, also, some limitations. First of all, the methodology presented in this work is supported by a single case study. Multiple case studies might be beneficial for pointing out the range of potential benefits of the methodology and demonstrating its robustness.

Secondly, this study addressed factors relevant for the specific application, which may not be generalized for different kinds of robot applications. Again, alternative studies within different areas of application might validate the suitability of the method for multiple areas or highlight the specific suitability for some areas rather than others.

To conclude, future research should extend and strengthen the methodology presented in this work by conducting further studies, addressing diversity in types of robots used, the structure of the activities and areas of applications.

\section{References}

Bartneck, C., Kulić, D., Croft, E. and Zoghbi, S. (2009), "Measurement instruments for the anthropomorphism, animacy, likeability, perceived intelligence, and perceived safety of robots", International Journal of Social Robotics, Vol. 1 No. 1, pp. 71-81.

Baxter, P., Kennedy, J., Senft, E., Lemaignan, S. and Belpaeme, T. (2016), "From characterizing three years of $\mathrm{HRI}$ to methodology and reporting recommendations", The Eleventh ACM/IEEE International Conference on Human Robot Interaction, IEEE Press, pp. 391-398.

Charisi, V., Davison, D., Reidsma, D. and Evers, V. (2016), "Children and robots: a preliminary review of methodological approaches in learning settings", Proceedings of the 2nd Workshop on Evaluating Child Robot Interaction at HRI, Christchurch, 7-10 March, available at: https://childrobotinteraction.org/ proceedings-2nd-workshop-hri-2016/

Eden, C. (2004), "Analyzing cognitive maps to help structure issues and problems", European Journal of Operations Research, Vol. 159 No. 3, pp. 673-686.

Feil-Seifer, D. and Matarić, M.J. (2011), "Automated detection and classification of positive vs. negative robot interactions with children with autism using distance-based features", HRI 2011-6th ACM/IEEE International Conference on Human-Robot Interaction, IEEE Press, pp. 323-330.

Frayling, C. (1993), "Research in art and design", Royal College of Art Research Papers, Vol. 1 No. 1, pp. 1-5.

Hanna, L., Risden, K., Czerwinski, M. and Alexander, K.J. (1998), "The role of usability research in designing children's computer products", The Design of Children's Technology, Morgan Kaufmann Publishers, Burlington, MA, pp. 3-26.

Huff, A.S. and Jenkins, M. (2002), Mapping Strategic Knowledge, Sage, London.

Kennedy, J., Baxter, P. and Belpaeme, T. (2015), "Can less be more? the impact of robot social behaviour on human learning", Proceedings of the 4th International Symposium on New Frontiers in Human and Robot Interaction, AISB-The Society for the Study of Artificial Intelligence and the Simulation of Behaviour, London.

Law, J. (2008), "Actor-network theory and material semiotics", in Turner, B.S. (Ed.), The New Blackwell Companion to Social Theory, Wiley-Blackwell, Oxford, pp. 141-158.

Lupetti, M.L. (2017), "Shybo-design of a research artefact for human-robot interaction studies", Journal of Science and Technology of the Arts, Vol. 9 No. 1, pp. 57-69.

Lupetti, M.L., Yao, Y., Mi, H. and Germak, C. (2017), "Design for children's playful learning with robots", Future Internet, Vol. 9 No. 3, pp. 1-20. Art. 52,

Norese, M.F. (2011), "An application of MACRAME to support communication and decisions in a multiunit project", Group Decision and Negotiation, Vol. 20 No. 1, pp. 115-131.

Norese, M.F. (2016), "A model-based process to improve robustness in multi criteria decision aiding interventions", Journal of Multi-Criteria Decision Analysis, Vol. 23 No. 5-6, pp. 183-196. 
Norese, M.F. and Salassa, F. (2014), "Structuring fragmented knowledge: a case study", Knowledge Management Research \& Practice, Vol. 12 No. 4, pp. 454-463.

Norese, M.F., Novello, C. and Salassa, F. (2015), "An integrated system to acquire knowledge and support decisions in complex innovation design processes", Journal of Organizational Computing and Electronic Commerce, Vol. 25 No. 2, pp. 194-212.

O'Keefe, R.M. (2016), "Experimental behavioural research in operational research: what we know and what we might come to know", European Journal of Operations Research, Vol. 249, pp. 899-907.

Read, J.C. and MacFarlane, S. (2006), "Using the fun toolkit and other survey methods to gather opinions in child computer interaction", Proceedings of the 2006 Conference on Interaction design and children, ACM, New York, NY, pp. 81-88.

Read, J.C., MacFarlane, S.J. and Casey, C. (2002), "Endurability, engagement and expectations: Measuring children's fun", Interaction Design and Children, Shaker Publishing, Eindhoven, Vol. 2, pp. 1-23.

Robert, D. and Breazeal, C. (2012), "Blended reality characters", HRI 2012-7th ACM/IEEE International Conference on Human-Robot Interaction, IEEE Press, pp. 359-366.

Roy, B. (1996), Multicriteria Methodology for Decision Aiding, Kluwer Academic, Dordrecht.

\section{Further reading}

Chen, S. and Epps, J. (2013), "Automatic classification of eye activity for cognitive load measurement with emotion interference", Computer Methods and Programs in Biomedicine, Vol. 110 No. 2, pp. 111-124.

Levialdi, S., Malizia, A., Onorati, T., Sangineto, E. and Sebe, N. (2007), "Detecting attention through telepresence", PRESENCE 2007-The 10th annual international workshop on Presence, ISBN: 978-09792217-1-2, pp. 233-236.

Maćkiewicz, M. and Cieciuch, J. (2016), "Pictorial personality traits questionnaire for children (PPTQ-C) a new measure of children's personality traits", Frontiers in Psychology, Vol. 7, Art. 498, pp. 1-11.

Mauss, I.B. and Robinson, M.D. (2009), "Measures of emotion: a review", Cognition \&Amp; Emotion, Vol. 23 No. 2, pp. 209-237.

Ros, R., Nalin, M., Wood, R., Baxter, P., Looije, R., Demiris, Y., Belpaeme, T., Giusti, A. and Pozzi, C. (2011), "Child-robot interaction in the wild: advice to the aspiring experimenter", Proceedings of the 13th international conference on multimodal interfaces, ACM, New York, NY, pp. 335-342.

\section{About the authors}

Maria Luce Lupetti is a Postdoctoral Researcher at Delft University of Technology. Previously, she was a PhD candidate at Politecnico di Torino, Italy. During the PhD, she was visiting scholar at X-Studio, Academy of Art and Design, of Tsinghua University, China. Maria Luce Lupetti is the corresponding author and can be contacted at: m.l.lupetti@tudelft.nl

Maria Franca Norese is an Associate Professor of Operations Research, at Politecnico di Torino. Her research interests are in the areas of multicriteria decision aiding, decision support systems and problem structuring methods, with a specific focus on systems and methodologies that facilitate understanding, collaboration and decision in organization. She has had articles published in several international journals.

Xiaolu Wu is a Master's Student in Management Engineering at Politecnico di Torino. As part of her Master thesis work, she is currently conducting research on cognitive mapping in cross-cultural contexts.

Haipeng $\mathrm{Mi}$ is Associate Professor at Tsinghua University (China) and co-director of X-Studio. Previously, he received a PhD from the University of Tokyo (Japan) where he was also assistant professor before joining Tsinghua.

For instructions on how to order reprints of this article, please visit our website: www.emeraldgrouppublishing.com/licensing/reprints.htm

Or contact us for further details: permissions@emeraldinsight.com 